Canad. Math. Bull. Vol. 22 (4), 1979

\title{
A NOTE ON A THEOREM OF KY FAN
}

\author{
BY \\ TZU-CHU LIN
}

Fan ([2, Theorem 2]) has proved the following theorem:

Let $K$ be a nonempty compact convex set in a normed linear space $X$. For any continuous map $f$ from $K$ into $X$, there exists a point $u \in K$ such that

$$
\|u-f(u)\|=\underset{x \in K}{\operatorname{Min}}\|x-f(u)\|
$$

In this note, we prove that the above theorem is true for a continuous condensing map defined on a closed ball in a Banach space. We also prove that it is true for a continuous condensing map defined on a closed convex bounded subset of a Hilbert space.

Now, we introduce our notations and definitions:

Let $B$ be a nonempty bounded subset of a metric space $X$. We shall denote (after Kuratowski [5]) by $a(B)$ the infimum of the numbers $r$ such that $B$ can be covered by a finite number of subsets of $X$ of diameter less than or equal to $r$.

Let $S$ be a nonempty subset of $X$ and let $f$ be a map from $S$ into $X$. If for every nonempty bounded subset $B$ of $S$ with $a(B)>0$, we have $a(f(B))<a(B)$, then $f$ will be called condensing ([7]). If there exists $k, 0 \leq k \leq 1$, such that for each nonempty bounded subset $B$ of $S$ we have $a(f(B)) \leq k a(B)$, then $f$ is called $k$-set-contractive ([5]).

Let $X, Y$ be two normed linear spaces, $S$ a nonempty subset of $X, f$ a map from $S$ into $Y, f$ is called nonexpansive if for each $x, y \in S$, we have $\|f(x)-f(y)\| \leq\|x-y\|$.

\section{LEMMA. ([4] or [7])}

Let $S$ be a nonempty closed convex bounded subset of a Banach space $X$. If $f$ is a continuous condensing map from $S$ into $S$, then $f$ has a fixed point in $S$.

THEOREM 1. Let $S$ be a closed ball with center at origin and radius $r$ in a $B$ anach space $X$. If $f$ is a continuous condensing map from $S$ into $X$, then there exists a point $u \in S$ such that

$$
\|u-f(u)\|=\underset{x \in \mathbf{S}}{\operatorname{Min}}\|x-f(u)\|
$$

Received by the editors December 7, 1977 and, in revised form, November 3, 1978. 
Proof. Define

$$
R(x)= \begin{cases}x, & \text { if }\|x\| \leq r \\ \frac{r x}{\|x\|}, & \text { if }\|x\| \geq r\end{cases}
$$

Then $R$ is a continuous 1 -set-contractive map ([6, Proposition 9]) from $X$ onto $S$. Define $F(x)=R(f(x)), F$ is a continuous map from $S$ into $S$. Moreover, for each nonempty bounded subset $B$ of $S$, with $a(B)>0$, we have

$$
a(F(B))=a(R(f(B))) \leq a(f(B))<a(B) .
$$

Thus $F$ is a condensing map. By Lemma, there exists $u \in S$ such that $F(u)=u$. Now,

$$
\begin{aligned}
\|u-f(u)\| & =\|F(u)-f(u)\|=\|R(f(u))-f(u)\| \\
& =\left\{\begin{array}{c}
\|f(u)-f(u)\|=0, \quad \text { if }\|f(u)\| \leq r \\
\left\|\frac{r f(u)}{\|f(u)\|}-f(u)\right\|=\|f(u)\|-r, \quad \text { if }\|f(u)\| \geq r
\end{array}\right.
\end{aligned}
$$

For each $x \in S$, we have $\|f(u)\|-r \leq\|f(u)\|-\|x\| \leq\|x-f(u)\|$ Hence

$$
\|u-f(u)\|=\underset{x \in S}{\operatorname{Min}}\|x-f(u)\| .
$$

THEOREM 2. Let $S$ be a nonempty closed convex subset of a Hilbert space $X$. Let $f$ be a continuous condensing map from $S$ into $X$. If $f(S)$ is bounded, then there exists a point $u \in S$ such that

$$
\|u-f(u)\|=\underset{x \in S}{\operatorname{Min}}\|x-f(u)\|
$$

Proof. By ([3]), there exists a continuous map $p$ from $X$ into $S$, such that for each $y \in X$, we have

$$
\|p(y)-y\|=\underset{x \in \mathbf{S}}{\operatorname{Min}}\|x-y\|
$$

By ([1]) $p$ is nonexpansive in Hilbert space. Then $p \circ f$ is a continuous condensing map from $c l c o p \circ f(S)$ (where clco A denote the closed convex hull of $A$ ) into clco $p \circ f(S)$. By Lemma, there exists $u \in S$ such that $p \circ f(u)=u$. Hence

$$
\|u-f(u)\|=\|p(f(u))-f(u)\|=\underset{x \in S}{\operatorname{Min}}\|x-f(u)\| .
$$

Remark. Only continuous map can not assure Theorem 1 is true. We use a well-known example to illustrate our case. Let $S$ be the closed unit ball in the Hilbert space $1_{2}$. Let

$$
f(x)=\left(\sqrt{ }\left(1-\|x\|^{2}\right), x_{1}, x_{2}, \ldots, x_{n}, \ldots\right)
$$


where $x=\left(x_{1}, x_{2}, \ldots, x_{n}, \ldots\right) \in S$. Since $\|f(x)\|=1$ therefore $f(S) \subset S$. If there were a point $u \in S$ such that

$$
\|u-f(u)\|=\underset{x \in S}{\operatorname{Min}}\|x-f(u)\| .
$$

it must be a fixed point of $f$. But it is easily seen that $f$ has no fixed point in $S$.

Added in proof. We can adopt the same technique used by F. E. Browder (On a sharpened form of the Schauder fixed-point theorem, Proc. Natl. Acad. Sci. U.S.A., Vol. 74, No. 11, pp. 4749-4751, November 1977) to obtain the following theorems by using our Theorem 1 and Theorem 2: The hypotheses are the same as Theorem 1 (or Theorem 2) if in addition, for each $x \in S$ with $x \neq f(x)$, there exists $y$ in $I_{S}(x)=\{x+c(z-x) \mid$ for some $z \in S$, some $c>0\}$ such that $\|y-f(x)\|<\|x-f(x)\|$, then $f$ has a fixed point in $S$. We also note that if $f$ is weakly inward (i.e. $f(x)$ lie in the closure of $I_{S}(x)$ for each $x \in S$ ), then for each $x \in S$ with $x \neq f(x)$ we can choose $y$ in $I_{S}(x)$ such that $\|y-f(x)\|<\|x-f(x)\|$.

Acknowledgement. This paper was written on Taiwan; the author is grateful to Professor Chi-Lin Yen for calling the author's attention to ([1]). On August 19, 1978, the author left for the United States. Now the author is a graduate student of the University of Iowa.

\section{REFERENCES}

1. Cheney, W. and Goldstein, A. A., Proximity maps for convex sets, Proc. Amer. Math. Soc. Vol. 10 (1959), 448-450.

2. Fan, K., Extensions of two fixed point theorems of F. E. Browder, Math. Z. 112 (1969), 234-240.

3. Fan, K. and Glicksberg, I., Some geometric properties of the sphere in a normed linear spaces, Duke Math. J. (1958), 553-568.

4. Furi, M. and Vignoli, A., On $\alpha$-nonexpansive mappings and fixed points, Atti. Accad. Naz. Lincei Rend. Cl. Sci. Fis. Mat. Natur. (8), Vol. 48 (1970), 195-198.

5. Kuratowski, C., Sur les espaces complets, Fund. Math. 15 (1930), 301-309.

6. Nussbaum, R. D., The fixed point index and fixed point theorems for $k$-set-contractions, doctoral dissertation, The univ. of Chicago, 1969.

7. Sadovski, B. N., A fixed point principle, Func. Anal. and Appl. 1 (1967), 151-153.

DEPARTMENT OF MATHEMATICS

UNIVERSITY OF IOWA

IOWA CITY

IOWA 52242 